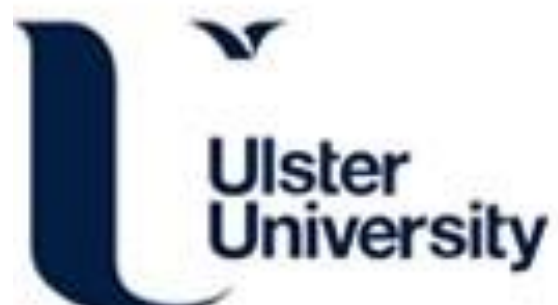

\section{Effects of vitamin D as a regulator of androgen intracrinology in LNCAP prostate cancer cells}

Cobice, D., Smith, K., Thompson, P., Rodriguez, E. P., \& Mackay, L. (2019). Effects of vitamin D as a regulator of androgen intracrinology in LNCAP prostate cancer cells. Biochemical and Biophysical Research Communications, 519(3), 579-584. https://doi.org/10.1016/j.bbrc.2019.09.059

Link to publication record in Ulster University Research Portal

\section{Published in:}

Biochemical and Biophysical Research Communications

\section{Publication Status:}

Published (in print/issue): 12/11/2019

DOI:

10.1016/j.bbrc.2019.09.059

\section{Document Version}

Author Accepted version

\section{General rights}

Copyright for the publications made accessible via Ulster University's Research Portal is retained by the author(s) and / or other copyright owners and it is a condition of accessing these publications that users recognise and abide by the legal requirements associated with these rights.

\section{Take down policy}

The Research Portal is Ulster University's institutional repository that provides access to Ulster's research outputs. Every effort has been made to ensure that content in the Research Portal does not infringe any person's rights, or applicable UK laws. If you discover content in the Research Portal that you believe breaches copyright or violates any law, please contact pure-support@ulster.ac.uk. 


\title{
Effects of Vitamin D as a Regulator of Androgen Intracrinology in LNCAP Prostate Cancer Cells
}

\author{
Karl W Smith ${ }^{1,2}$, Paul D Thompson², Edna Patricia Rodriguez², Logan Mackay³ \\ and Diego F Cobice ${ }^{1 *}$
}

${ }^{1}$ Mass spectrometry Centre, Biomedical Sciences Research Institute (BMSRI), School of Biomedical Sciences, Ulster University, Coleraine, Northern Ireland, UK

2 The Nutrition Innovation Centre for Food and Health (NICHE), Biomedical Sciences Research Institute (BMSRI), School of Biomedical Sciences, Ulster University, Coleraine, Northern Ireland, UK

${ }^{3}$ Scottish Instrumentation and Research Centre for Advanced Mass Spectrometry (SIRCAMS), EastChem School of Chemistry, University of Edinburgh, Scotland, UK

*Corresponding author: To Diego Cobice. d.cobice@ulster.ac.uk, tel: +442892604456. 


\section{Abstract}

Prostate cancer is initially treated via androgen deprivation therapy (ADT), a highly successful treatment in the initial pursuit of tumor regression, but commonly restricted by the eventual emergence of a more lethal 'castrate resistant' form of the disease. Intracrine pathways that utilize dehydroepiandrosterone (DHEA) or other circulatory precursor steroids, are thought to generate relevant levels of growth-stimulating androgens such as testosterone $(\mathrm{T})$ and dihydrotestosterone (DHT). In this study, we explored the capacity of the active vitamin $\mathrm{D}$ hormone to interact and elicit changes upon this prostatic intracrine pathway at a metabolic level. We used androgen dependent LNCaP cells cultured under steroid-depleted conditions and assessed the impact of vitamin D-based compounds upon intracrine pathways that convert exogenously added DHEA to relevant metabolites, through Mass Spectrometry (MS). Changes in relevant metabolism-related gene targets were also assessed. Our findings confirm that exposure to vitamin D based compounds, within LNCaP cells, elicits measurable and significant reduction in the intracrine conversion of DHEA to T, $\mathrm{DHT}$ and other intermediate metabolites within the androgenic pathway. The aassessment and validation of the biological model and analytical platforms were performed by pharmacological manipulations of the SRD5 $\alpha$ and HSD-17 $\beta$ enzymes. The data provides further confirmation for how a vitamin D-based regime may be used to counter intracrine mechanisms contributing to the emergence of castrate-resistant tumors.

\section{Keywords}

Prostate cancer

Metabolism

Vitamin D

Intracrinology

Mass Spectrometry 


\section{Abbreviations}

MALDI: Matrix Assisted Laser desorption ionization

FTICRMS: Fourier-transform ion cyclotron resonance mass spectrometry

T: Testosterone

DHT: Dihydrotestosterone

\section{Introduction}

Androgen deprivation therapy (ADT) is a treatment option for high risk, locally advanced or metastatic forms of prostate cancer $(\mathrm{PC})[1,2]$. It facilitates the reduction of bioavailable activating androgen receptor (AR) ligands, such as testosterone $(T)$ and dihydrotestosterone (DHT) [3] that promote tumor growth. The initial clinical responses to ADT are highly favorable but temporal, and often associated with relapse to a lethal castrate resistant form of the disease (CRPC) with a tumor phenotype that still retains an active AR-mediated pathway $[3,4]$, despite the low serum levels of T. Various mechanisms thought to underpin this recurrence have been proposed and reviewed that include; hypersensitivity through amplification or mutations within the AR gene [5-7], recruitment of co-activators, and altered steroidogenesis $[8,9]$. While these processes may each in part contribute to CRPC, there is increasing evidence that retained androgen signaling within the tumor may be significantly driven through uptake of circulating adrenal steroids, such as dehydroepiandrosterone (DHEA), and their subsequent intracrine conversion to AR-activating ligands [10,11]. Delineating the genetic changes and protein functions that drive such adaptive metabolic networks represents a challenge for the future development of therapeutic regimens based upon enzymatic inhibition and manipulated expression of encoding target genes $[9,12,13]$. While evidence support vitamin D may protect against prostate cancer initiation [14], its potential as a treatment for more aggressive forms of the disease and how it may interact with androgen pathways, is less clear. The vitamin D receptor (VDR) is 
appreciably expressed in prostate $[15,16]$ with the hormonally active form of vitamin $D_{3}$, $1 \alpha, 25$-dihydroxyvitamin- $D_{3}(1,25 \mathrm{D})$ shown to regulate the gene expression of components that may dictate the flux of steroid hormone metabolic pathways (Figure 1) [17]. Our group has previously described the capacity of ligand-activated VDR to enhance expression of the cytochrome P450 genes, CYP3A4 and CYP3A5, leading to an increased level of metabolic inactivation of testosterone and DHEA within LNCaP and LAPC-4 prostate cancer cells $[18,19]$. These CYP3A enzymes have been found to cause hydroxylation of steroidal compounds that include testosterone, DHEA, androstenedione (A4) and dihydrotestosterone (DHT) [20-22]. These findings indicate that the selective regulation of CYP3A and other metabolism-related genes through a vitamin D-based regimen, may represent a novel therapeutic approach that applied in combination with ADT, could enable simultaneous targeting of the bioavailability of both serum and tumoral androgens. This concept is supported through clinical studies that suggest patients with a higher level of CYP3A4 expression in prostate tissue have a more successful chance of cancer specific survival and lower levels of relapse PSA (prostate specific antigen), indicating towards a positive link with upregulation and cancer suppression [23].

In this study, we expand upon our previous reports and assess how ligand activated VDR within prostate cancer cells may impact upon the intracrine biosynthesis of androgens generated from exogenously derived DHEA. Through application of a mass spectrometry platform used in combination with specific enzymatic inhibitors and expression analysis of key metabolic genes, we assess the conversion of specific androgens within our metabolic system, and evaluate the potential mechanisms through which VDR-mediated effects modulate pathways that dictate the endogenous levels of activating AR ligands. 


\section{Materials and Methods}

\subsection{Cell culture and ligands}

LNCaP cell line were obtained from the European Collection of Authenticated Cell Cultures (LNCaP clone FGC (ECACC 89110211)) and maintained in RPMI 1640 with 10\% foetal bovine serum (FBS), 1\% Penicillin/Streptomycin and 1\% L-Glutamine (GIBCO, Invitrogen). For experiments using steroid depleted conditions, cells were supplemented with Phenol-red free RPMI (PRF-RPMI) with 5\% charcoal stripped serum (CSS) (GIBCO, Invitrogen). All cells were routinely maintained at $37^{\circ} \mathrm{C}$ with $5 \% \mathrm{CO}_{2}$. All nuclear receptor ligands were dissolved in ethanol (vehicle) and included; $1 \alpha, 25$ dihydroxyvitamin-D 3 (Sigma, UK), EB1089 (Tocris Bioscience, UK) and DHEA (TCI Europe). Hydroxydehydroepiandrosterone (mixisomers), testosterone, androstenedione, $5 \alpha$-androstanedione, dihydrotestosterone, hydroxytestosterone (mixisomers) were obtained from Steraloids (Newport, US). Enzymatic inhibitions were through application of ritonavir, kindly provided by Professor Andrew Owen, University of Liverpool, with Finasteride and 2,4-dihydroxybenzophenone and Girard Reagent purchased from Sigma, UK and Testosterone-2,3,4- ${ }^{13} \mathrm{C} 3$ from Cerilliant, USA.

\subsection{Cell Culture treatments}

LNCaP cells were seeded in standard growth media at $2 \times 10^{6}$ cells/flask (medium$\mathrm{T} 75 \mathrm{~cm}^{3}$ flask) and incubated at $37^{\circ} \mathrm{C}$ for $24 \mathrm{hr}$ to allow cell attachment. Media was then removed and replaced with PRF-RPMI \& 5\% CSS containing either 1,25D (10nM), EB1089 (10nM), or vehicle control. Following incubation for $24 \mathrm{hr}$ at $37^{\circ} \mathrm{C}$, media was replenished with CSS/PRF-RPMI containing vehicle with, 1,25D (10nM) or EB1089 $(10 \mathrm{nM})$ and/or DHEA $(100 \mu \mathrm{M})$ treatments. Cells were incubated for a further 24 hours, before media was collected for metabolomic analysis, with cells trypsinized and pelleted for subsequent gene expression analysis. For RTV treated cells, the same conditions were used above, with a 24-hour pre-treatment of vehicle (ethanol) or 1,25D (10nM) and 
then the addition of DHEA $(100 \mu \mathrm{M})$ in fresh media, but included addition of RTV (100nM) during DHEA incubation period, and media was collected for analysis after $24 \mathrm{hrs}$. Biological inhibitor assays involved $24 \mathrm{hr}$ incubation of cells in CSS/PRF-RPM, containing $100 \mu \mathrm{M}$ DHEA in combination with either finasteride $(10 \mu \mathrm{M}), 2,4-\mathrm{DBP}(2 \mu \mathrm{M})$ or vehicle control (DMSO), with media collected and analyzed.

\subsection{Steroid extraction and in-situ chemical derivatisation from cell media}

Media was removed from culture flasks at the specified time point after initiation of treatment and samples were extracted using Liquid-Liquid Extraction (LLE) with ethyl acetate. $750 \mu \mathrm{L}$ of centrifuged cell media was added to $250 \mu \mathrm{L}$ of water, $0.5 \mathrm{ml} 2 \mathrm{M} \mathrm{NaCl}$ and spiked with internal standard $\left(100 \mu \mathrm{M}\right.$ Testosterone-2,3,4- $\left.{ }^{13} \mathrm{C} 3\right)$. Then, $2 \mathrm{~mL}$ ethyl acetate was added, vortexed for 10 seconds and left to separate in an ice bath for 20 mins. An aliquot $(1 \mathrm{ml})$ of the supernatant was removed and dried down under $\mathrm{N}_{2}$ gas at RT. Samples were reconstituted with $100 \mu \mathrm{L}$ of Girard-T $(1 \mathrm{mg} / \mathrm{ml}$ in $\mathrm{MeOH})$ and $50 \mu \mathrm{L}$ of acetic acid, vortexed for $3 s$ and left on a heat block at $40^{\circ} \mathrm{C}$ for 60 mins. Then, $50 \mu \mathrm{L}$ of water was added after reaction completion. Samples were then transferred to an HPLC amber vial insert for analysis. Matrix preparation and spotting: Matrix was applied using the dried droplet technique. In brief, $50 \mu \mathrm{L}$ of the derivatized samples was mixed with $50 \mu \mathrm{L}$ of $\mathrm{CHCA}$ ( $\alpha$-Cyano-4-hydroxycinnamic acid) matrix $(10 \mathrm{mg} / \mathrm{ml}$ in $80 \%$ ACN with $0.1 \%$ TFA). Vortexed for $2 \mathrm{~s}$ and $1.0 \mu \mathrm{L}$ was spotted on a Bruker anchor MALDI target for Fourier Transform Ion Cyclotron Mass Spectrometry (FTICR-MS) analysis.

\subsection{Matrix Assisted Laser Desorption lonization coupled with High}

\section{Resolution Mass Spectrometry (MALDI-FTICR-MS) analysis of steroid metabolism}

Mass Spectrometry analysis was performed using a 12T SolariX MALDI- FTICR-MS (Bruker Daltonics, MA, US) employing a Smartbeam $1 \mathrm{kHz}$ laser, with instrument control using SolariX control v1.5.0 (build 42.8) was used. Ions were accumulated across 800 
laser shots and laser power was optimized for consistent ion production. lons were detected using constant accumulation of selected ions (CASI) in positive ion mode. Isolation windows were set at $(\mathrm{m} / z 415.1 \pm 50 \mathrm{Da})$ and $(\mathrm{m} / z 515 \pm 50 \mathrm{Da})$ to include all targeted Gir-T androgens derivatives with an isolation time of 120 s yielding a 4 Mword time-domain transient. Collision energies were optimized for optimal daughter ions production of ketosteroids Girard-T derivatives as outlined in Table S2. Mass calibration was performed using ${ }^{13} \mathrm{C}$ labelled testosterone derivative (ISTD) at $\mathrm{m} / \mathrm{z} 405.3270 \mathrm{using}$ single point correction algorithm. Processing was performed using Data Analysis 4.4 built (Bruker Daltonics, Bremen, Germany). The product ions were identified using an error tolerance of $5 \mathrm{ppm}$ for their corresponding theoretical monoisotopic masses. Data was reported as ratios as previously performed by Cobice et al. [24,25]. Fragmentation patterns were proposed and confirmed by accurate mass measurements (Figure S6).

\subsection{Statistical Analysis}

Data analysis for metabolite conversions was performed using SPSS (version 25), IBM,

US. Data are expressed as mean \pm SEM of three independent experiments, and differences were analyzed using one-way ANOVA with a Tukey post-hoc test. Statistical significance was accepted at $p<0.05$. Gene expression results were analyzed using a Microsoft Excel (version 16), using unpaired Student t-test. Statistical significance was accepted at $p<0.05$, or otherwise indicated. 


\section{Results}

\subsection{Activation of VDR within prostate cancer cells results in a reduced metabolic conversion of DHEA to Testosterone and \\ Dihydrotestosterone}

Mass spectrometry analysis was performed to quantitate changes within the CRPC androgen biosynthetic pathway within $\mathrm{LNCaP}$ cells that result from exposure to vitamin D-based treatments. Data depicted in Figure $\mathbf{2 a}$ revealed significant fluctuations in the 'flow' between components comprising this intracrine network. While conversion of DHEA to A4 is not affected by addition of VDR ligands, there is a significant increase in HydroxyDHEA detected between treatments. Exposure to 1,25D increases the production of HydroxyDHEA by approximately 2-fold, with EB1089 achieving a 6-fold elevation in the production of this metabolite, consistent with our previous studies [19]. Following the A4 conversion from DHEA (Figure 2b), differences were noted in the levels of testosterone and DHT. Induction of VDR within LNCaP cells using 1,25D or EB1089, resulted in a reduction the levels of testosterone by approximately $30 \%$ or $50 \%$ respectively. Similar effects of VDR activation were observed for the metabolic conversion of DHT, with the most significant effects found for EB1089 in lowering DHT to less than $50 \%$ of the levels detected in the control group. The levels of $5 \alpha$ androstanedione were also reduced with 1,25D and EB1089 achieving similar effects. In contrast, hydroxytestosterone was increased 2-fold following addition of VDR activating ligands, in accordance with our previous reports $[18,19]$.

To further explore a mechanistic basis, the impact of VDR ligand activation was investigated upon the expression of key metabolism-related genes (Figure S7 and Figure S8). This data shows the responsive changes in mRNA encoding CYP3A gene that follow in LNCaP cells cultured in the presence of DHEA, and VDR activation by addition of $1,25 \mathrm{D}$. In the presence of 1,25D, the expression of both CYP3A4 and CYP3A5 was elevated. However, upon combined treatment, strikingly opposing 
transcriptional responses were noted for both genes. This treatment achieved an increase in CYP3A4 expression but at a level that was reduced compared to $1,25 \mathrm{D}$ by itself. In contrast, DHEA shown a co-operative enhancement of CYP3A5 expression with

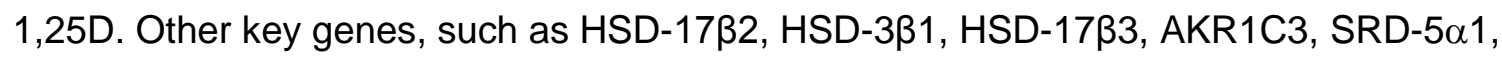
and SRD-5 $\alpha 2$ were assessed under the same liganded conditions for mRNA expression (Figure S8). Expression of HSD-17ß2 is key to note, as it was unresponsive to $1,25 \mathrm{D}$ as previously observed [26], but applied combination of 1,25D with DHEA resulted in a striking co-operative induction (Figure S8b).

\subsection{CYP3A enzyme activity significantly contributes to the metabolic inactivation of Testosterone and Dihydrotestosterone within prostate cancer cells}

Contribution of the specific encoding enzymatic function was also assessed upon the metabolic flux within the CRPC pathway as shown in Figure 3. A CYP3A enzyme inhibitor, ritonavir (RTV) was used. DHEA with and without RTV displayed no metabolic flux, except from a slight increase of A4, indicating RTV was directly involved at the initial steps of the CRPC metabolic pathway.

For A4 and hydroxy-DHEA, no significant changes were observed across treatments (Figure 3a). Data depicted within Figure 3b show that testosterone is largely affected by the addition of RTV and levels were lowered to vehicle (DHEA) upon addition of Vit D. Same trend was detected with DHT, showing significantly higher levels when RTV was added. Upon RTV and DHEA/VitD, DHT levels were significantly increased and level of hydroxylated testosterone were decreased in presence of either RTV or (RTV/VitD) treatments. 


\subsection{Assessment of biological model and analytical platforms by inhibition of SRD5 $\alpha$ and HSD-17 $\beta$ enzymes}

To validate biological and analytical model platforms, experiments involving addition of finasteride or 2,4-dihydroxybenzophenone (2,4-DBP) were performed. These compounds represent an established pharmacological manipulation of the CRPC intracrine pathway, targeting inhibition of SRD5 $\alpha$ and HSD-17ß3 enzymes, respectively. As shown in Figure 4, levels of A4 were significantly higher in samples containing either of the two inhibitors compared to DHEA treatment. T levels increased upon finasteride treatment against control ( $\sim 30 \%)$ and 2,4-DBP ( 50\%). Consequent to 2,4-DBP treatment, testosterone levels were decreased, and opposite effects were observed in 5a-dione. 5a-dione and DHT levels were significantly reduced with finasteride and both steroids increased upon 2,4-DBP treatment. Hydroxytestosterone follows a mild trend with its testosterone precursor, as both finasteride and 2,4-DBP treated cells levels were increased. 


\section{Discussion}

Here, the effects of ligand activated VDR upon metabolic pathways are studied within LNCaP prostate cancer cells, with specific focus on the metabolic intracrine conversions of exogenously added DHEA. DHEA is considered the main circulatory precursor contributing to the synthesis of testosterone (T) and dihydrotestosterone (DHT) [27]. While LNCaP cells are considered androgen-dependent, they also harbor a mutated form of AR that can be activated by DHEA and other metabolites within the androgen biosynthetic pathway [28] and therefore used in this study as representative of tumor phenotypes that potentially respond to adrenal sourced and intracrine-derived compounds during of the course of ADT. Our previous reports have detailed intracrinerelated effects of vitamin D within a range of prostate cancer (LNCaP, LAPC4, PC3, DU145) and non-tumorigenic prostate cells (RWPE1) which collectively stress such VDR-mediated effects are requisite upon co-expression of $A R[18,19]$, highlighting intriguing interactions between the vitamin $\mathrm{D}$ and androgen hormonal pathways. We have demonstrated that ligand-activated VDR within LNCaP cells has significant impact upon the intracrine conversion of DHEA to androgen-based metabolites.

This was established by using a MALDI-FTICR-MS analytical platform developed in this study as a high throughput assay. The ultrahigh resolution of FTICR-MS in combination with collision induced dissociation (CID) and Isotopic Fine Structure (IFS) features provided high-confidence molecular formulas assignments for targeted androgens, resulting in unmatched accuracy for compound identification without the need of chromatography separation (Figure S2-S5). The use of CID/IFS in combination of Girard T derivatization provided unique selectivity as fragmentation can distinguish between $\mathrm{A}$ and $\mathrm{D}$ ring keto steroids via six- or five-member ring rearrangements of the hydrazine moiety respectively (Figure S6).

In this study, VDR activation is also shown to diminish levels of the primary AR activating 
ligands T and DHT (Figure 2 and 3). Both 1,25D and EB1089 treatments also significantly reduced the levels of $5 a$-dione, $\mathrm{T}$ and $\mathrm{DHT}$, while increasing the levels of the inactive hydroxylated form of T. Several downstream effects of VDR activation potentially explain these observations. VDR mediates the increased expressions of CYP3A4 and CYP3A5, as displayed in Figure S7, leading to increased oxidative inactivation and levels of the biologically inactive hydroxylated forms of T and DHEA, confirming our previous observations [19]. Such hydroxylation events of one or more of these steroids may result in overall reduced levels of interconversion and metabolic 'flux' within the CRPC pathway. The reduced bioavailability of androgens to initiate AR mediated responses may contribute to prevent disease relapse and emergence of an ADTresistant tumor $[29,30]$. The co-operative upregulation of HSD-17ß2 following exposure to both $1,25 \mathrm{D}$ and DHEA (Figure S8) may reflect activation of both AR and VDR activation that leads to a favored conversion of T and DHT to A4 and $5 \alpha$-dione, respectively, will partly drive the pathway in a desired direction away from the potent ligands. Chang et al has shown that the CRPC pathway follows the 'DHT backdoor pathway' (DHEA $\rightarrow \mathrm{A} 4 \rightarrow 5 \alpha$-dione $\rightarrow \mathrm{DHT}$ ) with $5 \alpha$-dione being the main driver of growth for prostate cancer cell lines, when dosed with A4 against T [10]. In this study, the focus area was narrowed to the reactions highlighted in Figure $\mathbf{1}$ as steroidogenesis is extensive and multi levelled, with various enzyme families involved. We have suggested that the reduced levels of $T$ that result from 1,25D treatment can largely be attributed to generation of hydroxylated T or the interconversion of other metabolites within the CRPC. Lundgvist et al 2011 however also described an up-regulation of CYP19A1 (aromatase) mRNA within LNCaP cells in response to $1,25 \mathrm{D}$. This may imply that a proportion of the testosterone involved in our system may also be converted to estradiol within our experiments [17]. Due to the complexity of steroidogenesis within prostatic cancer cells, many intermediate and further conversion metabolites may influence the fluctuation of measured analytes. 
Finally, we have applied enzyme inhibitors finasteride and 2,4-dihydroxybenzophenone (2,4-DBP) (Figure 4) to demonstrate the system we used produced an expected alteration of the steroid metabolites. Finasteride was used to inhibit the SRD5 $1 / 2$ enzyme and 2,4-DBP for the inhibition HSD-17ß3, and so partially altering the levels of the relevant interconvertible targeted androgens. Advanced progression of prostate cancer to a 'castrate resistant' form is complex, with multi-interacting networks contributing to the emergence and continued growth of the tumor. While current ADT approaches may not realize a long term and absolute depletion of tumoral androgens or AR activating steroids, our current data provide further support for the co-application of a vitamin D-based regime as a means to counter contribution of intracrine pathways towards disease relapse.

\section{Acknowledgments/Funding}

This work was supported by The Department for Employment and Learning for Northern Ireland and Internal Research Challenge Fund from Ulster University. 


\section{References}

[1] C. Huggins, R.E. Stevens, C.V. Hodges, Studies on prostatic cancer: II. The effects of castration on advanced carcinoma of the prostate gland, Archives of surgery 43 (1941) 209223.

[2] P. Abedinpour, V.T. Baron, J. Welsh, P. Borgström, Regression of prostate tumors upon combination of hormone ablation therapy and celecoxib in vivo, The Prostate 71 (2011) 813823.

[3] J.L. Mohler, C.W. Gregory, O.H. Ford, D. Kim, C.M. Weaver, P. Petrusz, E.M. Wilson, F.S. French, The androgen axis in recurrent prostate cancer, Clinical cancer research 10 (2004) 440-448.

[4] J.A. Locke, E.S. Guns, A.A. Lubik, H.H. Adomat, S.C. Hendy, C.A. Wood, S.L. Ettinger, M.E. Gleave, C.C. Nelson, Androgen levels increase by intratumoral de novo steroidogenesis during progression of castration-resistant prostate cancer, Cancer research 68 (2008) 6407-6415.

[5] T. Visakorpi, E. Hyytinen, P. Koivisto, M. Tanner, R. Keinänen, C. Palmberg, A. Palotie, T. Tammela, J. Isola, O.-P. Kallioniemi, In vivo amplification of the androgen receptor gene and progression of human prostate cancer, Nature genetics 9 (1995) 401.

[6] C.W. Gregory, R.T. Johnson, J.L. Mohler, F.S. French, E.M. Wilson, Androgen receptor stabilization in recurrent prostate cancer is associated with hypersensitivity to low androgen, Cancer research 61 (2001) 2892-2898.

[7] E. Jernberg, A. Bergh, P. Wikström, Clinical relevance of androgen receptor alterations in prostate cancer, Endocrine connections 6 (2017) R146-R161.

[8] J. Kumagai, J. Hofland, S. Erkens-Schulze, N.F.J. Dits, J. Steenbergen, G. Jenster, Y. Homma, F.H. de Jong, W.M. van Weerden, Intratumoral conversion of adrenal androgen precursors drives androgen receptor-activated cell growth in prostate cancer more potently than de novo steroidogenesis, The Prostate 73 (2013) 1636-1650. 
[9] T. Chandrasekar, J.C. Yang, A.C. Gao, C.P. Evans, Mechanisms of resistance in castration-resistant prostate cancer (CRPC), Translational andrology and urology 4 (2015) 365.

[10] K.H. Chang, R. Li, M. Papari-Zareei, L. Watumull, Y.D. Zhao, R.J. Auchus, N. Sharifi, Dihydrotestosterone synthesis bypasses testosterone to drive castration-resistant prostate cancer, Proceedings of the National Academy of Sciences 108 (2011) 13728-13733.

[11] Q. Mo, S.-f. Lu, N.G. Simon, Dehydroepiandrosterone and its metabolites: differential effects on androgen receptor trafficking and transcriptional activity, The Journal of steroid biochemistry and molecular biology 99 (2006) 50-58.

[12] R.M. Attar, C.H. Takimoto, M.M. Gottardis, Castration-resistant prostate cancer: locking up the molecular escape routes, Clinical Cancer Research 15 (2009) 3251-3255.

[13] S.K. Pal, J. Patel, M. He, B. Foulk, K. Kraft, D.A. Smirnov, P. Twardowski, M. Kortylewski, V. Bhargava, J.O. Jones, Identification of mechanisms of resistance to treatment with abiraterone acetate or enzalutamide in patients with castration-resistant prostate cancer (CRPC), Cancer 124 (2018) 1216-1224.

[14] A.V. Krishnan, D.L. Trump, C.S. Johnson, D. Feldman, The role of vitamin D in cancer prevention and treatment, Rheumatic Disease Clinics 38 (2012) 161-178.

[15] R.J. Skowronski, D.M. Peehl, D. Feldman, Vitamin D and prostate cancer: 1, 25 dihydroxyvitamin D3 receptors and actions in human prostate cancer cell lines, Endocrinology 132 (1993) 1952-1960.

[16] M.R. Haussler, G.K. Whitfield, C.A. Haussler, J.C. Hsieh, P.D. Thompson, S.H. Selznick, C.E. Dominguez, P.W. Jurutka, The nuclear vitamin D receptor: biological and molecular regulatory properties revealed, Journal of Bone and Mineral Research 13 (1998) 325-349.

[17] J. Lundqvist, M. Norlin, K. Wikvall, 1a, 25-Dihydroxyvitamin D 3 exerts tissue-specific effects on estrogen and androgen metabolism, Biochimica et Biophysica Acta (BBA)Molecular and Cell Biology of Lipids 1811 (2011) 263-270. 
[18] D. Doherty, S.A. Dvorkin, E.P. Rodriguez, P.D. Thompson, Vitamin D receptor agonist EB1089 is a potent regulator of prostatic "intracrine" metabolism, The Prostate 74 (2014) 273-285.

[19] O. Maguire, C. Pollock, P. Martin, A. Owen, T. Smyth, D. Doherty, M.J. Campbell, S. McClean, P. Thompson, Regulation of CYP3A4 and CYP3A5 expression and modulation of "intracrine" metabolism of androgens in prostate cells by liganded vitamin D receptor, Molecular and cellular endocrinology 364 (2012) 54-64.

[20] Q. Cheng, C.D. Sohl, F.K. Yoshimoto, F.P. Guengerich, Oxidation of dihydrotestosterone by human cytochromes P450 19A1 and 3A4, Journal of Biological Chemistry (2012) jbc-M112.

[21] K.K.M. Miller, J. Cai, S.L. Ripp, W.M. Pierce, T.H. Rushmore, R.A. Prough, Stereo-and regioselectivity account for the diversity of dehydroepiandrosterone (DHEA) metabolites produced by liver microsomal cytochromes P450, Drug Metabolism and Disposition 32 (2004) 305-313.

[22] S. Ohmori, H. Nakasa, K. Asanome, Y. Kurose, I. Ishii, M. Hosokawa, M. Kitada, Differential catalytic properties in metabolism of endogenous and exogenous substrates among CYP3A enzymes expressed in COS-7 cells, Biochimica et Biophysica Acta (BBA)General Subjects 1380 (1998) 297-304.

[23] T. Fujimura, S. Takahashi, T. Urano, J. Kumagai, T. Murata, K. Takayama, T. Ogushi, K. Horie-Inoue, Y. Ouchi, T. Kitamura, Expression of cytochrome P450 3A4 and its clinical significance in human prostate cancer, Urology 74 (2009) 391-397.

[24] D.F. Cobice, D.E.W. Livingstone, A. McBride, C.L. MacKay, B.R. Walker, S.P. Webster, R. Andrew, Quantification of $11 \beta$-hydroxysteroid dehydrogenase 1 kinetics and pharmacodynamic effects of inhibitors in brain using mass spectrometry imaging and stableisotope tracers in mice, Biochemical pharmacology 148 (2018) 88-99.

[25] D.F. Cobice, C.L. Mackay, R.J.A. Goodwin, A. McBride, P.R. Langridge-Smith, S.P. Webster, B.R. Walker, R. Andrew, Mass spectrometry imaging for dissecting steroid intracrinology within target tissues, Analytical chemistry 85 (2013) 11576-11584. 
[26] J.-H. Wang, P. Tuohimaa, Regulation of $17 \beta$-hydroxysteroid dehydrogenase type 2 , type 4 and type 5 by calcitriol, LXR agonist and $5 \alpha$-dihydrotestosterone in human prostate cancer cells, The Journal of steroid biochemistry and molecular biology 107 (2007) 100-105. [27] C.K. Tsao, M.D. Galsky, A.C. Small, T. Yee, W.K. Oh, Targeting the androgen receptor signalling axis in castration-resistant prostate cancer (CRPC), BJU international 110 (2012) $1580-1588$.

[28] J. Veldscholte, C. Ris-Stalpers, G. Kuiper, G. Jenster, C. Berrevoets, E. Claassen, H.C.J. Van Rooij, J. Trapman, A.O. Brinkmann, E. Mulder, A mutation in the ligand binding domain of the androgen receptor of human LNCaP cells affects steroid binding characteristics and response to anti-androgens, Biochemical and biophysical research communications 173 (1990) 534-540.

[29] C.M. Armstrong, A.C. Gao, Adaptive pathways and emerging strategies overcoming treatment resistance in castration resistant prostate cancer, Asian journal of urology 3 (2016) 185-194.

[30] R.J. Auchus, The backdoor pathway to dihydrotestosterone, Trends in Endocrinology \& Metabolism 15 (2004) 432-438. 


\section{Figure legends:}

Figure 1: Main Castrate resistant prostate cancer (CRPC) androgen pathway. DHEA: Dehydroepiandrosterone, T: Testosterone, A4: Androstenedione, 5a-dione: $5 \alpha$ Androstanedione, DHT: Dihydrotestosterone.

Figure 2: Activation of VDR within prostate cancer cells results in a reduced metabolic conversion of DHEA to Testosterone and Dihydrotestosterone. LNCaP cells were treated within steroid free media with vehicle control, $10 \mathrm{nM} 1,25 \mathrm{D}$ or $10 \mathrm{nM}$ EB1089 for a 24-hour treatment period. After the initial 24-hour period, media was refreshed with the addition of the VDR ligand treatments and $100 \mu \mathrm{M}$ of DHEA was introduced to the cell media for a steroid induced environment for a further 24-hour period. Data reveal that in comparison to control groups, treatment with $1,25 \mathrm{D}$ or the synthetic agonist EB1089 elicits significant fluctuations in the 'flow' between components comprising in this intracrine network. a) Conversion percentage levels of DHEA to A4 by addition of VDR ligands. b) As A4 is a superior precursor of the CRPC pathway following the initial reaction, other metabolites of the pathway are expressed as a conversion from A4. The major conversion differences are noted in the levels of testosterone and DHT. Data are mean \pm SEM. $p<0.05^{\star}, p<0.01^{* \star}, p<0.001^{* \star *}$.

Figure 3: Metabolic effect of the activation and inhibition of CYP3A enzymes.

LNCaP cells were treated with vehicle, 10nM 1,25D, 100nM RTV or 10nM 1,25D and 100nM RTV for 24 hours in steroid depleted media. The following 24-hour period introduced the same treatments and $100 \mu \mathrm{M}$ DHEA for a steroid induced environment. Media from this experiment was derivatized as previously described in section $\mathbf{2 . 4}$. a) Percentage level conversion of A4 and hydroxy-DHEA from DHEA b) Metabolic conversion levels of CRPC steroids from A4. Data are mean \pm SEM. $p<0.05^{\star}, p<0.01^{* *}$, $p<0.001^{* * *}$. 
Figure 4: Assessment of biological model and analytical platforms by inhibition of SRD5 $\alpha$ and HSD-17 $\beta$ enzymes. LNCaP cells were treated using known enzyme inhibitors of HSD-17ß3 (2,4-dihydroxybenzophenone) and SRD5 $\alpha$ enzymes (finasteride). Under steroid free conditions for 24 hours, LNCaP cells were allowed to grow, then treated with vehicle control, $10 \mu \mathrm{M}$ finasteride or $2 \mu \mathrm{M} 2,4-\mathrm{DBP}$, along with each of the treatment sets being dosed with $100 \mu \mathrm{M}$ DHEA for a further 24 -hour period. a) Conversion levels of A4 are shown from the precursor DHEA b) Other steroid metabolites are displayed in conversion percentage from A4. Data are mean \pm SEM. $p<0.05^{\star}, p<0.01^{* *}, p<0.001^{* * *}$ 
Figure 1: Smith et al., 2019
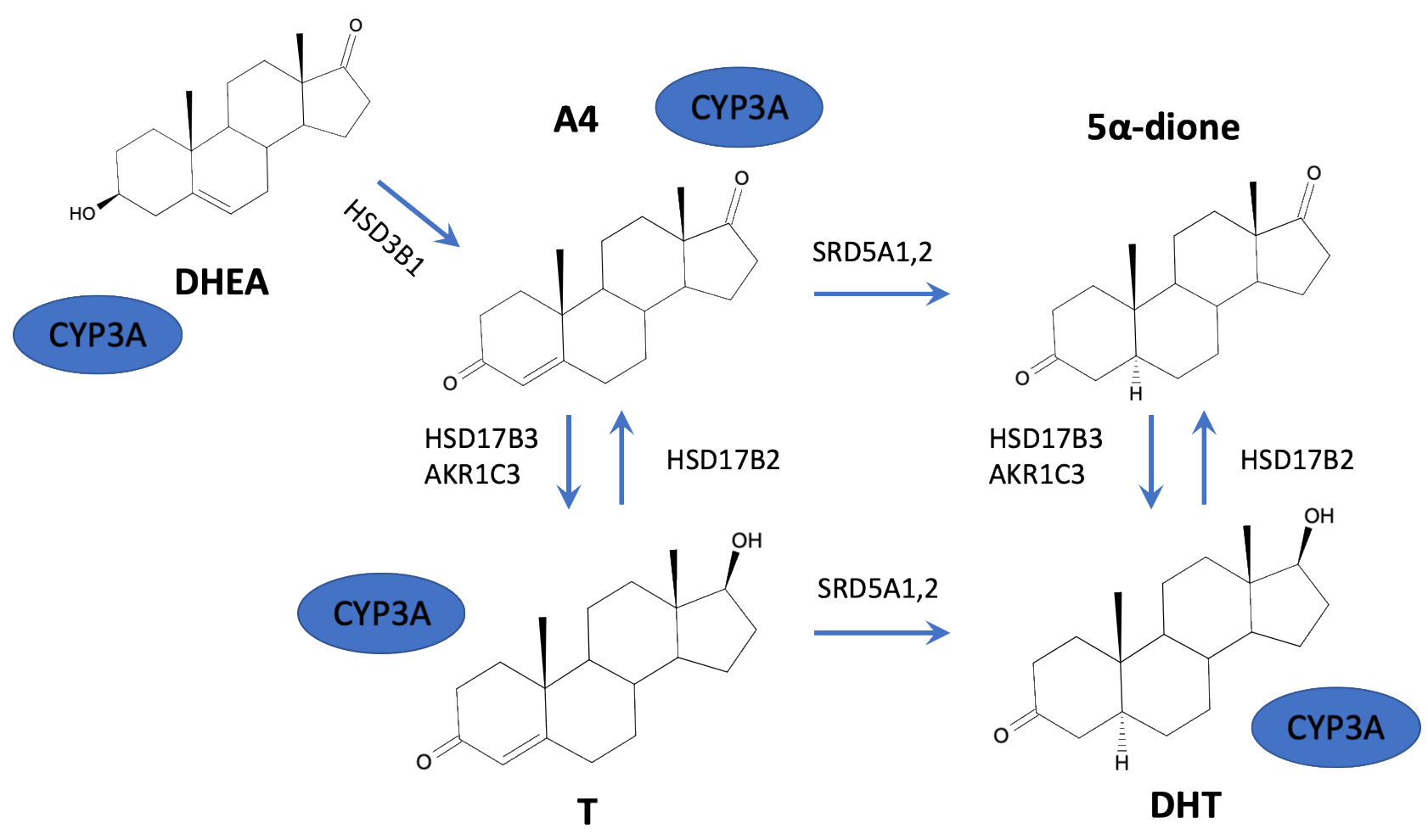

Figure 2: Smith et al., 2019
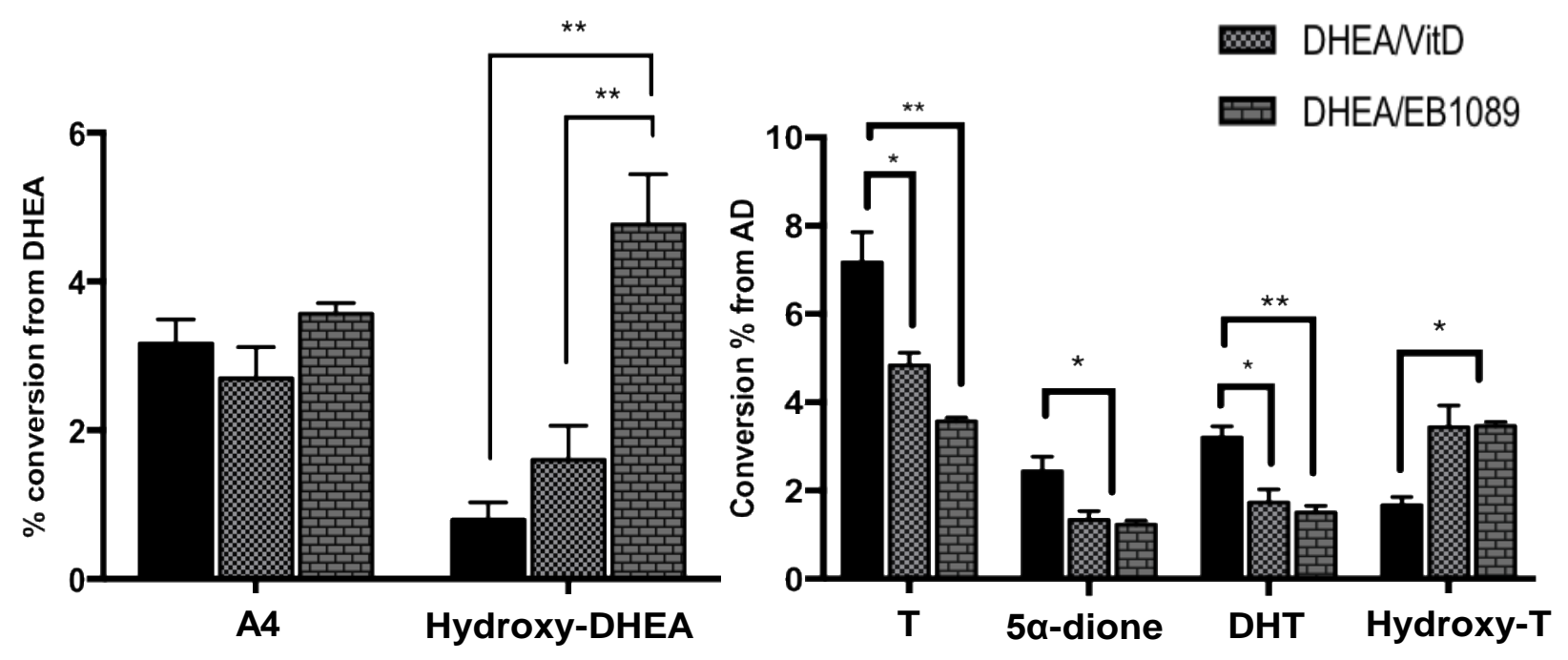
Figure 3: Smith et al., 2019
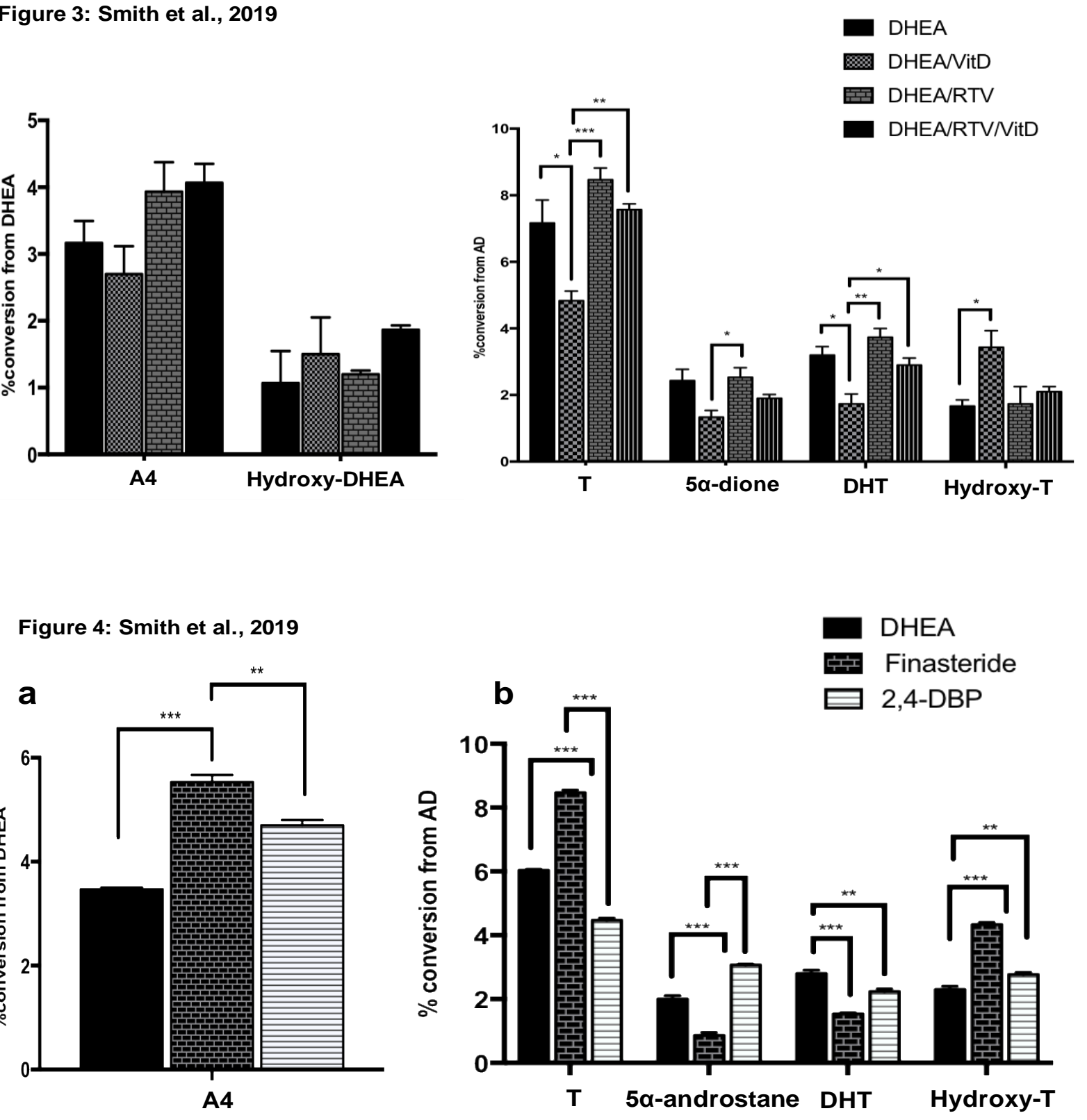